\section{Dedifferentiation in glioma}

Neural stem cells (NSCs) are thought to be the cell of origin in glioblastoma multiforme (GBM), an aggressive brain tumor in humans. Now, Inder Verma and colleagues suggest that dedifferentiation of neurons and astrocytes into an NSC or progenitor state can induce gliomas in a mouse model (Science, published online 18 October 2012; doi:10.1126/ science.1226929). The authors established a mouse glioma model with Cre-inducible lentiviral vectors expressing short hairpin RNAs (shRNAs) for Nf1 and Tp53, as it has been shown previously that loss of these factors leads to the formation of high-grade gliomas. Injection of the vectors into Synapsin I-Cre mice, in which they should target neurons but not glial cells, resulted in glioma formation. The authors then targeted more mature neurons by injecting CamK2a-Cre mice with the Creinducible lentiviral vectors, which led to the development of tumors but with a much longer latency, suggesting that mature neurons may be more refractory to transformation. The authors found that, as the tumors progressed, expression of progenitor/ stem cell markers increased, while expression of differentiated state markers decreased. The authors then transformed primary cortical astrocytes in vitro and transplanted them into immunodeficient mice. The resulting tumors also expressed progenitor markers, showing that terminally differentiated astrocytes undergo dedifferentiation during tumorigenesis.

\section{Circadian genomics}

The mammalian circadian clock is composed of an autoregulatory network in which the BMAL1, CLOCK, NPAS2, PER and CRY transcription factors interact in transcriptional feedback loops. Although it is known that the circadian clock regulates the expression of thousands of transcripts, Joseph Takahashi and colleagues now report the genome-wide architecture of the transcriptional network regulated by the circadian clock in mammals (Science 338, 349-354, 2012). The authors used chromatin immunoprecipitation sequencing (ChIP-seq) to profile the DNA binding of BMAL1, CLOCK, NPAS2, PER1, PER2, CRY1, CRY2, RNA polymerase 2, p300 and CBP throughout the circadian cycle in mouse liver. They also measured gene expression with RNA sequencing. They found circadiandependent binding of transcription factors and RNA polymerase 2 and circadian-dependent variation in RNA expression. They also profiled the histone modifications $\mathrm{H} 3 \mathrm{~K} 4 \mathrm{me} 1, \mathrm{H} 3 \mathrm{~K} 4 \mathrm{me} 3, \mathrm{H} 3 \mathrm{~K} 9 \mathrm{ac}$, $\mathrm{H} 3 \mathrm{~K} 27 \mathrm{ac}, \mathrm{H} 3 \mathrm{~K} 36 \mathrm{me} 3$ and $\mathrm{H} 3 \mathrm{~K} 79 \mathrm{me} 2$ across the genome throughout the circadian cycle using ChIP-seq. They found strong circadiandependent patterns of RNA polymerase 2 recruitment and $\mathrm{H} 3 \mathrm{~K} 4 \mathrm{me} 3$, $\mathrm{H} 3 \mathrm{~K} 9 \mathrm{ac}$ and $\mathrm{H} 3 \mathrm{~K} 27 \mathrm{ac}$ occupancy at transcriptional start sites, also finding evidence for circadian-dependent histone modifications at the majority of expressed genes.

\section{RNA decay QTL}

Expression quantitative trait loci (eQTLs) are genetic variants associated with variation in steady-state gene expression levels. Now, Athma Pai, Jonathan Pritchard, Yoav Gilad and colleagues report a study that

Written by Pamela Feliciano, Emily Niemitz \& Kyle Vogan identifies QTLs associated with mRNA decay rates (PLoS Genet. 8, e1003000, 2012). The authors determine the gene expression decay rates of over 16,000 genes in 70 human lymphoblastoid cell lines from the HapMap Project for which there is whole-genome genotype data available. To measure decay rates, they treated cells with an RNA elongation complex inhibitor, actinomycin $\mathrm{D}$, and measured changes in mRNA abundance at various time points after treatment. They observed both variation in decay rates between genes and variation in decay rates between individuals within the same gene. They mapped genetic variation associated with the quantitative trait of mRNA decay rate and found 195 loci, called RNA decay QTLs (rdQTLs). The authors estimate that the expression of $10 \%$ of genes is influenced by variation in decay rates and that $\mathrm{mRNA}$ decay rates contribute to the mechanism of a substantial fraction of eQTLs.

$E N$

\section{Resistant to cancer}

Blind mole rats (BMRs) of the genus Spalax are subterranean rodents that are long-lived for their size and are cancer resistant. Now, Andrei Seluanov and colleagues suggest that cancer resistance in BMRs is mediated by a concerted necrotic cell death mechanism (Proc. Natl. Acad. Sci. USA, published online 5 November 2012; doi:10.1073/pnas.1217211109). The authors cultured primary BMR fibroblasts and found that the cultures underwent concerted cell death (CCD) after about $3 \mathrm{~d}$. Flow cytometry analysis showed that cell death was mostly necrotic rather than apoptotic. The authors then tested whether CCD is regulated by the major tumor suppressor pathways, $\mathrm{Rb}$ and $\mathrm{p} 53$. They transfected BMR cells with SV40 large T antigen, which inactivates both $\mathrm{p} 53$ and $\mathrm{Rb}$, and found that cells proliferated continuously and did not undergo CCD; however, if only p53 or only $\mathrm{Rb}$ was inactivated, $\mathrm{CCD}$ was not rescued. The authors hypothesized that CCD was a response to interferon (IFN). Young BMR cultures did not release IFN, but older cultures secreted IFN- $\beta$ just before the onset of CCD. Notably, cultures rescued by CCD did not release IFN- $\beta$. The authors suggest that $\mathrm{CCD}$ protects BMR cells against cancer, and future experiments should test whether CCD can protect BMRs from chemical induction of cancer.

\section{Hh signal transduction in cilia}

Primary cilia are known to be key sites of Hedgehog (Hh) signal transduction in vertebrate cells, but the underlying molecular mechanisms are poorly understood. Rajat Rohatgi and colleagues now identify a new ciliary subdomain called the EvC zone that has an important role in transducing Hh signals (Dev. Cell 23, 823-835, 2012). The authors show that the products of the EVC and EVC2 genes, which are disrupted in the human ciliopathies Ellis-van Creveld syndrome and Weyers acrofacial dysostosis, colocalize at a region of the ciliary membrane immediately adjacent to the transition zone. They further show that diseasecausing mutations that result in Evc2 mislocalization throughout the entire ciliary membrane disrupt $\mathrm{Hh}$ signaling downstream of Smoothened (Smo). At the biochemical level, they observe that Smo forms a complex with Evc and Evc2 in response to Hh pathway stimulation. On the basis of their findings, the authors propose that interactions between Smo and the Evc-Evc2 complex at the EvC zone facilitate the critical signaling events leading to the cilia-dependent activation of Gli transcription factors in response to $\mathrm{Hh}$ signal. 